\title{
The Role of Quercetin in Ameliorating ARDS in COVID-19 Patients
}

\author{
Saurabh Sharma, DO, MS and Anand Seth*, PhD \\ SK Patent Associates LLC, Dublin, Ohio, USA
}

\begin{abstract}
The sudden outbreak of a novel coronavirus in December 2019 in Wuhan, China, known as Covid-19, spread throughout the world as a global pandemic. An immediate search started throughout the world to combat the virus on various fronts. Many clinical trials were initiated and are underway to utilize the existing drugs to delineate and combat virulence of the virus. Several vaccines have been developed and are under development including various combinations of herbal remedies. Molecular docking studies have shown that bioactive compounds like Quercetin found in medicinal plants have the best potential to act main protease inhibitor which in turn can help fight the virus. Quercetin improves arterial blood gas, lung water content, protein content, and cell count in bronchoalveolar lavage (BAL). ARDS-related mortality is high among Covid-19 patients. Quercetin can potentially serve as a potent supplement for the group of people who are infected with Corona-19 virus and predisposed to acute respiratory distress syndrome (ARDS), ARDS is one of the major causes of mortality in SARS-CoV-2 / Covid-19 infected patients.
\end{abstract}

\section{Introduction}

\section{COVID-19 and ARDS}

December 2019 was the first time a case of COVID-19 was identified in Wuhan, China, and ever since the virus has spread throughout the globe-spanning 222 countries with over more than $5,477,405$ deaths worldwide as of 24 January 5, 2022. Clinically SARS-CoV-2 can manifest as disorders of cardiovascular, GI, neurological, and pulmonary systems. However, the most concerning complication of the virus is an acute hypercapnic respiratory failure requiring noninvasive positive pressure ventilation like BiPAP and in more severe cases invasive mechanical ventilation [1]. Sometimes acute hypoxemia can lead to a very severe acute lung injury and manifest as acute respiratory distress syndrome (ARDS). ARDS continues to be a major cause of mortality in intensive care units. Although mechanical ventilation with low Tidal Volume, early neuromuscular blockage, and prone positioning have shown to reduce mortality in ARDS, there is no specific treatment available [2]. Pathophysiology of ARDS involves diffuse alveolar damage due to the formation of hyaline membranes, increased capillary permeability, interstitial edema, and an influx of circulating inflammatory cells. Although wide evidence of research has shown the role of neutrophil influx and its activation within the lungs as important factors in the pathogenesis of ARDS, alveolar macrophages (AMs) and alveolar and bronchial epithelial cells are also involved $[3,4]$. Studies have shown that AMs play a role in modulation of the inflammatory responses and resultant lung injury $[3,4]$. AMs secrete pro-inflammatory cytokines, including TNF-alpha, IL-1 beta, and IL- 6 which in turn stimulate neutrophils. Activated neutrophils release oxidants, proteases, leukotrienes, and platelet-activating factors resulting in the development of ARDS.

\section{Flavonoid-Quercetin}

Flavonoids are antioxidants. They scavenge particles in the body called free radical which are known to damage cell membranes, damage DNA, and cause cell death. Quercetin is one of the most abundant dietary flavonoids and is found in a broad range of fruits, vegetables, seeds, nuts, flowers, barks, broccoli, olive oil, green tea, red grapes, red wine, dark cherries, and berries like blueberries and cranberries. The highest concentration of flavonoids is found in vegetables like onion and broccoli, fruits like apples, cherries, and berries, and drinks like tea and red wine [5]. It is known to have antiviral, anti-inflammatory, cytotoxic, antimicrobial, and antioxidant effects. Studies have shown that regular diet provides small amounts of quercetin ( $<1 \mathrm{uM}$ ) which is not sufficient to exhibit chemo-preventative and/or protective effects on various organ systems like the cardiovascular system. However, the levels can be increased to appropriate plasma levels (> 10uM)

*Corresponding author: Anand Seth, SK Patent Associates LLC, Dublin, Ohio, USA

Accepted: December 29, 2021

Published online: December 31, 2021

Citation: Sharma S, Seth A (2021) The Role of Quercetin in Ameliorating ARDS in COVID-19 Patients. Ann Public Health Reports 5(2):246-248 
by appropriate quercetin enriched foods or supplements [6]. Quercetin suppresses $\mathrm{H}_{2} \mathrm{O}_{2}$-induced apoptotic events, including hypodiploid cells, activation of Caspase 3 enzyme activity, and lactate dehydrogenase release [2]. In vitro studies have shown that Quercetin plays a role in protecting against allergies, hay fever, heart disease, high cholesterol, HTN, interstitial cystitis, prostatitis, RA, and cancer.

\section{Quercetin and COVID-19}

COVID-19 is a highly infectious virus and can lead to comorbidities especially ARDS. The onset of COVID-19 related ARDS is typically 8-12 days, which is inconsistent with ARDS Berlin criteria, which defined a 1-week onset limit [7]. Studies have shown that quercetin plays a role in diminishing an acute lung injury, nitric oxide (NO), and malondialdehyde (MDA), and increases the antioxidant enzyme levels [8]. Animal sepsis models using cecal ligation and puncture have shown that single-dose of quercetin diminishes levels of YKL40 (an inflammatory glycoprotein involved in endothelial dysfunction), and oxidant molecules (oxidase (XO)) [3]. Takashima, et al. [9] have shown that quercetin exhibits a prophylactic effect on lipopolysaccharide (LPS)-induced acute lung injury (ALI) in mice [9]. Lipopolysaccharides are present in the membrane of gram-negative bacteria and is considered as one of the major risk factors for ARDS. The same study also showed that intrathecal administration is a desirable method to effectively exert the cytoprotective effects of quercetin. Hayashi, et al. [10] showed that quercetin increases the levels of heme oxygenase (HO-1) expression and protects against hydrogen peroxide $\left(\mathrm{H}_{2} \mathrm{O}_{2}\right)$-induced cytotoxicity in lung epithelial cell lines [10]. Peroxide induced cytotoxicity is one of the etiologies of acute lung injury and in very severe forms can lead to ARDS. HO-1 plays an important role in defense against oxidant-induced lung injury during ARDS. Studies have shown increased HO-1 expression in lung tissues and bronchoalveolar lavage (BAL) fluid from patients with ARDS as body's compensatory role to fight against cytotoxicity. Several studies on various murine models of lung injury have also shown a protective role for $\mathrm{HO}-1$.

Researchers have elucidated numerous antiviral properties of flavonoids. Molecular docking studies have shown that bioactive compounds found in medicinal plants like naringenin, quercetin, apigenin-7-glucoside, oleuropein, curcumin, catechin, and epicatechin-gallate appeared to have the best potential to act as COVID-19 main protease $\left(\mathrm{M}_{\text {pro }}\right)$ inhibitors as demonstrated by the binding energies [11]. This was also supported by a very recent study using in silico molecular dynamics docking studies by Peterson, et al [12]. In another docking study, it was shown that SARS-CoV2 has a favorable binding affinity for the human ACE2 receptor, which facilitates infection and molecules like quercetin can serve as a reasonable target to disrupt the viral S-protein -ACE2 interface [13]. The study by Meng, et al. [14] showed that quercetin improved arterial blood gas, lung water content, protein content, and cell counts in BAL in a dose-dependent manner [9]. It also decreased the expression of the intercellular adhesion molecule (ICAM-1) and macrophage inflammatory protein (MIP-2) as compared to the control groups. Soluble intercellular cell adhesion molecule -1 (sICAM-1) levels are significantly higher in both the pulmonary edema fluid and plasma from patients with ALI/ARDS [2].

\section{Conclusion}

Therefore, numerous studies have elucidated that quercetin holds strong therapeutic potential in suppressing the inflammatory cascade in acute lung injury and ARDS. Bioflavonoids, in general, and in particular quercetin hold a strong potential to be used in synergistic forms along with antivirals. The effective synergism of flavonoids with antivirals could potentially serve as a potent prophylactic supplement for the vaccinated and unvaccinated populations.

\section{Competing Interests}

The authors declare no competing interests.

\section{Acknowledgement}

Thanks to Brinly Richards for assembling the information pertaining to the content of the paper.

\section{References}

1. Wilcox Susan R (2020) Management of respiratory failure due to COVID-19. BMJ 369: m1786.

2. Calfee CS, Eisner MD, Parsons PE, et al. (2009) Soluble intercellular adhesion molecule-1 and clinical outcomes in patients with acute lung injury 2009. Intensive Care Med 35: 248-257.

3. Matthay MA, Zemans RL (2011) The acute respiratory distress syndrome: Pathogenesis and treatment. Annu Rev Pathol 28: 147-163.

4. Ware LB, Matthay MA (2000) The acute respiratory distress syndrome. N Eng J Med 342: 1334-1349.

5. David AVA, Arulmolo R, Parasuram S (2016) Overviews of the biological importance of quercetin: A bioactive flavonoid. Pharmacogen Rev 10: 84-89.

6. Russo M, Spagnuolo C, Tedesco I, et al. (2012) The flavonoid quercetin in disease prevention and therapy: Facts and fancies. Biochem Pharmacol 83: 6-15.

7. Li X, Ma X (2020) Acute respiratory failure in COVID-19: Is it "typical" ARDS? Crit Care 24: 198.

8. Gerin F, Sener U, Erman H, et al. (2016) The effect of quercetin on acute lung injury and biomarkers of inflammation and oxidative stress in the rat model of sepsis. Inflammation 29: 700-705.

9. Takashima K, Matsushima M, Hashimoto K, et al. (2014) Protective effects of intratracheally administered quercetin on lipopolysaccharide-induced acute lung injury. Respir Res 15: 15.

10. Hayashi Y, Matudhima M, Nakamura T, et al. (2012) Quercetin protects against pulmonary oxidant stress via heme oxygenase-1 induction in lung epithelial cells. Biochem Biophys Res Commun 417: $169-174$.

11. Khaerunnisa S, Kurniawan H, Awaluddin R, et al. (2020) Potnetial inhibitor of COVID-19 main protease (Mpro) from several medicinal plant compounds by molecular docking study. Preprint 2020030226.

12. Peterson Leif (2020) COVID-19 and flavonoids: In silico molecular dynamics docking to the active catalytic site of SARSCoV and SARS-CoV-2 main protease. 
13. Smith M, Smith JC (2020) Repurposing therapeutics for COVID-19: Supercomputer-Based docking to the SARSCoV-2 viral spike protein and viral spike protein-Human ACE2 interface. Chem Rxiv.
14. Meng Lijing, Zhengyi Ly, Zhuoying Yu, et al. (2016) Protective effect of quercetin on acute lung injury in rats with sepis and its influence on ICAM-1 and MIP-2 expression. Genet Mol 15.

DOI: $10.36959 / 856 / 526$ 